\title{
Directed approach responses and positive conditioned suppression in the rat
}

\author{
JOHN KARPICKE \\ Indiana University, Bloomington, Indiana 47401
}

\begin{abstract}
Three experiments evaluated an alternative to accounts of positive conditioned suppression that stress central (i.e., motivational or emotional) states. This "competing-response" interpretation was tested by analyzing directed movements that develop in rats during a visual or an auditory stimulus (CS) that signals an appetitive reinforcer (US) in a situation where the subject is also emitting an instrumental response for food. In each experiment, positive conditioned suppression (i.e., a reduction in the rate of such instrumental responding during CS presentations) was accompanied by responses directed toward the CS source and/or the US-delivery site. In Experiment 1, a diffuse (auditory) CS signaled a US delivered at some specific place in the chamber and rats approached the US-delivery site during CS. In Experiments 2 and 3, the spatial proximity of a localized visual CS and US-delivery site determined whether CS-directed or US-directed behavior predominated during the CS. The results suggest that the topographies of conditioned responses on any positive conditioned suppression procedure depend upon the spatial arrangements of features that elicit and support these behaviors. They further suggest that the identification of these features and their spatial arrangements is necessary for the analysis of appetitive classical-instrumental interactions.
\end{abstract}

Many studies of interactions between classical and instrumental conditioning involve presenting a light or tone (CS), immediately followed by a primary reinforcer (US), to an animal performing a wellestablished instrumental response. Repeated responseindependent CS-US pairings usually result in the suppression of appetitively motivated responding during CS, whether the US is aversive (e.g., Estes \& Skinner, 1941) or appetitive (e.g., Azrin \& Hake, 1969). Azrin and Hake (1969) labeled the reductions in instrumental responding during preappetitive and preaversive CSs "positive" and "negative" conditioned suppression, respectively.

Several theoretical accounts of classical-instrumental interactions (Millenson \& deVilliers, 1972; Rescorla

The experiments reported here are a portion of a $\mathrm{PhD}$ thesis submitted to Indiana University, and were supported by National Institute of Mental Health Grant MH-19300 to E. Hearst. This manuscript was prepared while the author was a National Institutes of Health Postdoctoral Fellow in the Psychobiology Research Group at The Florida State University. Experiment 2 was reported at the meetings of the Eastern Psychological Association, New York City, 1976. I thank my committee chairman and advisor, Dr. E. Hearst, and the members of my thesis committee, Drs. J. A. Dinsmoor, C. G. Mueller, D. E. Robinson, and W. J. Rowland, for their help and encouragement. I also thank Drs. R. A. Boakes and G. Christoph for stimulating discussions of positive conditioned suppression and related matters. Alida Evans and Dexter Gormley deserve special thanks for their valuable technical advice and assistance. Requests for reprints of this article should be addressed to the author, Department of Psychology, Valparaiso University, Valparaiso, Indiana 46383.
\& Solomon, 1967) have emphasized the motivational properties of classically conditioned responses. According to this general type of explanation for conditioned suppression, an emotional or motivational state appropriate to the US becomes conditioned to the $\mathrm{CS}$ and disrupts appetitive behavior by somehow altering or competing with appetitive motivation. There is some evidence that is consistent with this general interpretation for negative conditioned suppression. Actual measurements of "emotional responses" (e.g., changes in heart rate or blood pressure) during positive conditioned suppression have, however, provided little or no support for it (Kelly, 1973).

A more peripheralistic account of positive conditioned suppression in rats was offered by Karpicke, Christoph, Peterson, and Hearst (1977; see LoLordo, McMillan, \& Riley, 1974; Schwartz, 1976, for similar analyses in pigeons). Karpicke et al. monitored their rats' positions in a standard instrumental training apparatus immediately preceding and during visual CSs located either several inches (far) from the instrumental-response manipulandum or very near it. These measures showed that CS-approach responses could be used to account for the larger amount of positive conditioned suppression which occurred with the far CS: Approach to the far CS apparently interfered more with the animals' ongoing instrumental behavior than did approach toward the CS near the manipulandum. The occurrence of these CS-approach responses is consistent with recent 
studies of the classical conditioning of unrestrained animals (see review by Hearst \& Jenkins, 1974). These studies have shown that directed (approach and contact) behaviors frequently develop toward localized CSs that signal appetitive USs.

Responses directed toward the CS source were not the only behaviors that Karpicke et al. found during positive conditioned suppression. Their rats also approached the US-delivery site more during the CS than at other times. This type of approach behavior (US approach) has been studied in rats in an autoshaping paradigm (i.e., with localized preappetitive CSs and US-delivery sites but no baseline of instrumental responding) by Boakes (1977). In his experiments, rats also approached both the CS source and the US-delivery site during CS presentations. In a similar experiment, Holland (1977) found large amounts of US-approach behavior during a diffuse auditory preappetitive CS. These studies suggest that US-approach behaviors could account for positive conditioned suppression during CSs that have no specific apparatus location. However, other investigators (Azrin \& Hake, 1969; Leslie \& Millenson, 1973) have, either made gross observations of their rats or measured contacts with their US-delivery device and have not found evidence for this type of behavior during positive conditioned suppression.

\section{EXPERIMENT 1}

Experiment 1 employed a diffuse auditory CS on two positive conditioned suppression procedures to determine whether US-approach responses would emerge during the CS and interfere with instrumental responding. Procedures for two groups of rats differed with respect to the spatial arrangements of the delivery locations for instrumental reinforcers and USs. The different arrangements of delivery sites allowed manipulation of the compatibility of possible US-approach responses with the pattern of behavior (pressing the lever and approaching the instrumental-reinforcer delivery site) maintained by the instrumental conditioning procedure.

One group of rats received response-contingent and CS-contingent milk deliveries from the same delivery device, while a second group had two milkdelivery devices, one for deliveries which followed leverpresses and the other for those following CSs. If US-approach behaviors are involved in positive conditioned suppression, rats in both groups should direct more behavior toward the US-delivery site during the CS than during an equal period of time preceding each CS. Moreover, the differential incompatibility of US-approach behavior with ongoing instrumental response sequences on these arrangements may produce more positive conditioned suppression on the two-dipper than on the one-dipper procedure.

\section{Method}

Subjects. Eight 90- to 100-day-old experimentally naive, male hooded rats, maintained at $80 \%$ of their ad-lib weights, served in two groups of four subjects each.

Apparatus. The front panel of a standard $29.0 \times 23.5 \times$ $19.5 \mathrm{~cm}$ rat chamber (Grason-Stadler E3125D) contained two $5.5-\mathrm{cm}$-diam milk-delivery apertures at grid floor level. One aperture was at the extreme left side of this panel, centered approximately $4 \mathrm{~cm}$ from the left wall. The other aperture was centered the same distance from the right wall. Each aperture had a $1.3-\mathrm{cm}$ diam hole in the bottom of it, through which a 1 - $\mathrm{ml}$ dipper-cup of milk (Borden's Eagle Brand sweetened condensed milk mixed with an equal volume of tap water) could be delivered. A transparent Plexiglas flap ("door") hung in front of the right aperture. A force of at least $.05 \mathrm{~N}$ would push the door fully open, but even slight movements of the door (openings as small as $2 \mathrm{~mm}$ ) were detected by a photoelectric circuit. The left aperture, which did not serve as a US-delivery site, was not equipped with a Plexiglas door

Because not all behaviors directed toward the US-delivery site would necessarily open the aperture door, touch sensors were connected to the chamber's floor grids to provide an additional measure of US approach. The 21 equally spaced $.32-\mathrm{cm}$-diam grids were perpendicular to the panel containing the apertures and were connected together to form three electrically isolated floor sections. The seven grids on the right third of the floor, in front of the US-delivery aperture, formed one such section and were connected to the "operate" lead of a drinkometer circuit. The chamber walls and the seven grids in the center of the floor were connected to the "common" lead of this circuit. Simultaneous observation of the rats and the sensor control unit showed that contacts of the right third of the floor and time spent on this floor section were quite accurately monitored by this arrangement.

A Gerbrands (G6312) lever was centered on the panel containing the apertures and $8 \mathrm{~cm}$ above the floor. Leverpresses of at least $.35 \mathrm{~N}$ counted as responses. Continuous dim chamber illumination was provided by a $2.5-\mathrm{cm}$-diam white-jeweled $(3.75-\mathrm{W})$ lamp fixture approximately $4 \mathrm{~cm}$ above the lever. A speaker, mounted on one inside wall of the ventilated Grason-Stadler sound-attenuating enclosure which housed the chamber, was located approximately $20 \mathrm{~cm}$ behind the front panel. It delivered either 80-dB SPL white noise or an $80-\mathrm{dB}$ SPL tone (nominally $1,000 \mathrm{~Hz}$ ) produced by a Foringer AU902 audio generator. White noise was continuous in the absence of CS, but was switched off when the tone CS was presented.

Electromechanical programming and recording equipment was located in an adjoining room.

Procedure. Each rat was shaped to press the lever for either 3 -sec access to milk at the right aperture (four rats) or 3-sec access to milk at the left aperture (four rats). The average time between reinforced leverpresses was gradually increased to $2 \mathrm{~min}$ (VI $2 \mathrm{~min}$, range $=20-320 \mathrm{sec}$ ) over six daily 45-min sessions. After three sessions on the VI 2-min schedule, the four rats that received milk at the left aperture were trained to obtain milk at the right aperture. For this 30-min dipper-training session, the lever was removed and the left aperture was covered. A single 3-sec operation of the right dipper occurred about every $30 \mathrm{sec}$ throughout this session.

With the lever replaced and both apertures uncovered, each group next received 3 days of training (CS-only phase) during which 10 daily 15 -sec tone presentations occurred on an average of one every $4 \mathrm{~min}$ (range $=2-6 \mathrm{~min}$ ). For 15 subsequent days (CS-US pairings phase), each CS presentation was immediately followed by three successive operations of the milk dipper to the right of the lever. Thus, one group (RR) received VI-milk and milk USs at the right aperture, while the other group (LR) received VI-milk at the left aperture and milk USs at the right aperture.

For all subjects the total numbers of leverpresses and instrumental reinforcers were recorded each day. In addition, during 
each session of the CS-only and CS-US pairings phases, the following measures were taken during the 15 -sec pre-CS and CS periods: leverpresses, discrete US approaches (openings of the Plexiglas door), the amount of time the aperture door was held open, and the amount of time each rat spent contacting the right side of the floor.

\section{Results and Discussion}

For each day, behavior ratios were computed for leverpresses and US approaches from the formula $A \div(A+B)$, where $A$ and $B$ represent the measures of behavior during CS and pre-CS periods, respectively. When behavior is unaffected by CS presentations, this ratio has a value of .50 . Values less than .50 reflect suppression of behavior during CS relative to pre-CS, whereas values greater than .50 reflect facilitation of behavior during CS as compared to pre-CS.

Mean US-approach ratios (based on discrete aperture entries) and leverpress ratios for both groups are shown in Figure 1. During the CS-only phase, behaviors were either little or not consistently affected by CS presentations. When ratios were averaged over the 3 days of this phase, none of them differed significantly from $.50[\operatorname{ts}(3)<2.96]$. Similarly, there was little effect of CS presentation on leverpressing or on aperture entries during the first day of the CS-US pairings phase. However, with continued CS-US pairings, leverpress ratios decreased and USapproach ratios increased. The US-approach ratios for LR subjects were consistently higher than those for RR subjects. When these ratios were averaged over the last three CS-US pairings sessions, there was no overlap between the groups [LR mean = $.85, \mathrm{RR}$ mean $=.64 ; \mathrm{t}(6)=4.28, \mathrm{p}<.01$ ]. Ratios computed from the other measures of US approach (aperture-entry duration, time on the right side of the chamber) and averaged over the last 3 days of this phase showed the same result: LR rats had mean

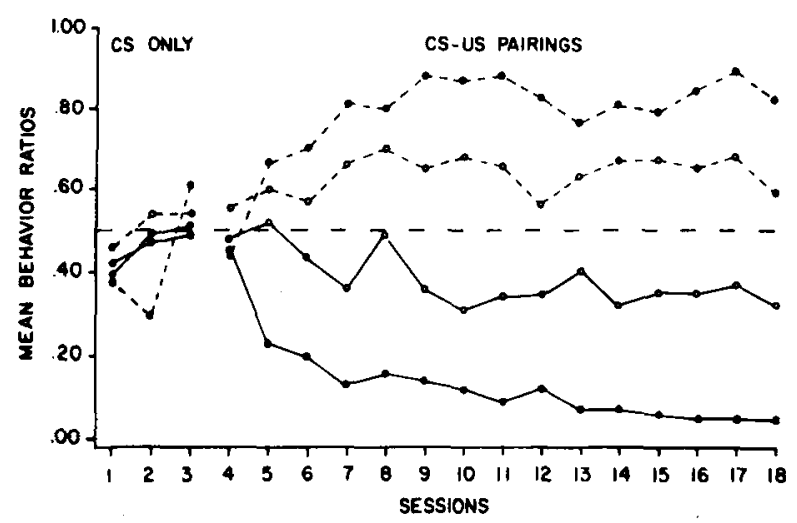

Figure 1. Mean leverpress and US-approach (aperture-entry) ratios for two-dipper (LR, filled circles) and one-dipper (RR, open circles) subjects during the CS-only and CS-US pairings phases of Experiment 1. Data points for leverpress ratios are connected by solid lines; broken lines connect data points for USapproach ratios.

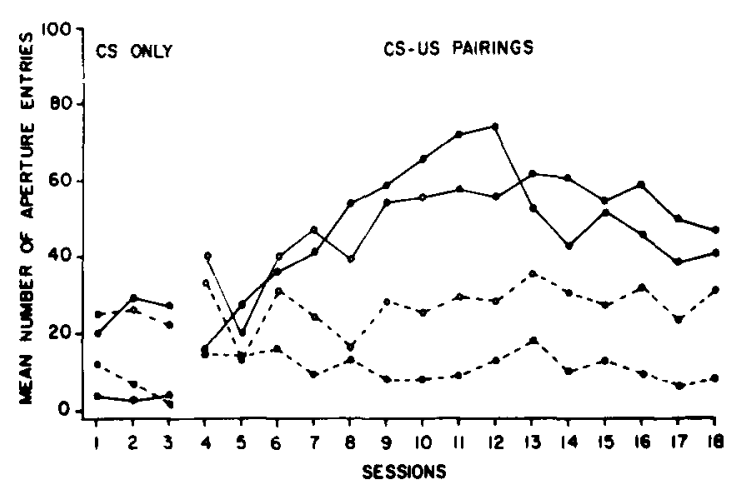

Figure 2. Mean numbers of US-aperture entries for two-dipper (LR, filled circles) and one-dipper (RR, open circles) subjects during the CS-only and CS-US pairings phases of Experiment 1. Data points for aperture entries during pre-CS periods are connected by broken lines; solid lines connect data points for aperture entries during $\mathrm{CS}$ periods.

US-approach ratios of .81 on the time-on-the-right measure and .87 on the aperture-entry-duration measure, and their individual scores did not overlap with those of $R R$ rats, which had respective means of .55 and .53 on these measures $[\operatorname{ts}(6)<5.50$, ps $<.01]$.

Leverpress ratios also differed on the two arrangements. There was no overlap between these ratios for the LR and RR subjects over the last three CS-US pairings sessions. Their respective mean suppression ratios were .05 and $.35[t(6)=2.90, p<.05]$; for these days, rats in both groups pressed the lever at essentially the same rate during pre-CS $(t<1.0)$.

Mean suppression and US-approach ratios for the last three CS-US pairings sessions were also compared to a .50 baseline ratio. Only the LR rats showed significant suppression of leverpressing [LR, $t(3)=$ $19.3, \mathrm{p}<.001 ; \mathrm{RR}, \mathrm{t}(3)=1.61]$, significant facilitation of time spent contacting the right side of the floor [LR, $\mathrm{t}(3)=3.77, \mathrm{p}<.05 ; \mathrm{RR}, \mathrm{t}(3)=1.22]$, and significant facilitation of time spent holding the aperture door open $[\mathrm{LR}, \mathrm{t}(3)=13.40, \mathrm{p}<.001$; $R R, t<1.0]$. However, discrete aperture entries were facilitated in both groups [ts $(3)>7.42$, ps $<.01]$. This latter result suggests that subjects in both groups learned about the CS-US relationship.

A more detailed analysis suggests that differential incompatibility of US-directed behaviors with ongoing instrumental response sequences accounts for the between-group suppression ratio differences. As shown in Figure 2, the difference between USapproach ratios for the two groups resulted from different amounts of behavior directed at the USdelivery site during pre-CS rather than CS periods. The mean numbers of daily dipper-entries for the last 3 days of training differed significantly for $R R$ vs. LR rats during pre-CS $[27.8$ vs. $7.3 ; \mathrm{t}(6)=3.64$, $\mathrm{p}<.02]$ but not during CS $(50.8$ vs. $40.8 ; \mathrm{t}<1.0)$. The same pattern of results was found for the other measures of US approach on these days: RR and LR 
subjects spent about the same portions of each $15-\mathrm{sec}$ $\mathrm{CS}$ on the right side of the chamber [RR mean = $11.4 \mathrm{sec}, \mathrm{LR}$ mean $=12.1 \mathrm{sec}, \mathrm{t}(6)=1.16]$ and similar amounts of time holding the aperture door open during CS [RR mean $=7.7 \mathrm{sec}$, LR mean $=11.4 \mathrm{sec}$, $\mathrm{t}(6)=1.93]$. However, during 15 -sec pre-CS periods, RR rats spent more time contacting the right side of the floor than did LR rats $[9.6 \mathrm{sec}$ vs. $2.9 \mathrm{sec}, \mathrm{t}(6)=$ $4.42, \mathrm{p}<.01]$ and more time at the right aperture [6.5 sec vs. $1.8 \mathrm{sec}, \mathrm{t}(6)=3.05, \mathrm{p}<.05$ ].

It is important to note that the small amounts of pre-CS time LR rats spent contacting the right side of the floor indicate that these subjects' intertrial behaviors were confined mainly to the left and center sections of the chamber. Gross observations of these subjects revealed that between trials they directed their behaviors toward the lever and the left aperture. Observations of RR subjects showed that they spent most of the intertrial time on the right and center sections of the chamber, pressing the lever and approaching the right aperture. This pattern of behavior is reflected in their higher (as compared to LR rats) pre-CS time-on-the-right and aperture-entry scores. During the CS, however, subjects in both groups directed their behaviors toward the right aperture. These approach responses were incompatible with the intertrial behaviors of LR subjects, and it is reasonable to suggest that their ongoing pattern of instrumental behavior was therefore severely disrupted. Over the last 3 days of training, they spent an average of $2.9 \mathrm{sec}$ during each pre-CS period and $12.1 \mathrm{sec}$ during each CS period contacting the right floor section $[t(3)=17.19, p<.001]$. On the other hand, approaches to the right aperture were actually part of the intertrial behavior pattern of RR subjects, and US approaches during CS might not be expected to severely disrupt their ongoing behavior. Even though discrete aperture entries increased significantly during CS relative to pre-CS for these subjects, over the last 3 days of training they spent little more CS than pre-CS time contacting the right side of the floor [11.4 vs. $9.6 \mathrm{sec}, \mathrm{t}(3)=1.52]$ or holding the aperture door open $(7.7$ vs. $6.5 \mathrm{sec}, \mathrm{t}<1.0)$. Thus, greater interference from US-approach behavior with instrumentally maintained response sequences in $\mathbf{L R}$ as compared to $R R$ rats seems a reasonable account for the greater positive conditioned suppression in the former group.

\section{EXPERIMENT 2}

Even though Experiment 1 and the results of Karpicke et al. (1977) have implicated directed skeletal responses in positive conditioned suppression, it is not clear whether CS-approach or US-approach responses would predominate on procedures that employ a localized CS source and a US-delivery device that requires approach and contact responses. Karpicke et al. reported the development of both responses on one of their arrangements of CS and manipulandum. However, the number of possible spatial arrangements of CS sources, US-delivery sites, and manipulanda within any given apparatus is large, and different arrangements might produce differential conditioning of one or the other type of response.

Two very simple arrangements of a localized CSsource and US-delivery site were employed in Experiment 2: The CS source and the US-delivery device were located either close to each other (on the same side of the manipulandum), or far apart (one on either side of the manipulandum). With these arrangements, CS-approach and US-approach responses were either compatible or incompatible with one another. Behaviors directed toward the CS source and toward the US-delivery site were recorded to determine which response would predominate on these two levels of CS-US proximity.

\section{Method}

Subjects. Eight experimentally naive 110 - to 120-day-old male hooded rats, maintained at $80 \%$ of their ad-lib weights, served in two groups of four subjects each.

Apparatus. The standard apparatus used in Experiment 1 was modified in several respects. The front panel contained only one $5.5-\mathrm{cm}-$ diam aperture, positioned at the extreme right, as in Experiment 1. Two $5.7 \times 1.3 \times 1.3 \mathrm{~cm}$ Plexiglas bars, wrapped with chromel wire, served as localized CS sources. The bars extended $1.3 \mathrm{~cm}$ into the chamber on the left and right sides of the front panel, and were centered about $3.5 \mathrm{~cm}$ from the side walls and $7 \mathrm{~cm}$ above the floor. Thus, the right $\mathrm{CS}$ source was positioned directly $(1.5 \mathrm{~cm})$ above the milk-delivery aperture. During CS periods, one or the other bar was lighted by supplying $14-\mathrm{V}$ dc to two $28-\mathrm{V}$ lamps within it. A chain manipulandum, which replaced the lever used in Experiment 1, hung midway between the CS sources; chainpulls of at least $.35 \mathrm{~N}$ counted as responses. The distance between the chain and the closest edge of either bar was about $5.5 \mathrm{~cm}$.

Dim chamber illumination was provided by a white-jeweled 2.5-cm-diam (3.75-W) lamp fixture centered on the front panel about $12 \mathrm{~cm}$ above the floor. A 75-dB SPL continuous white noise within the chamber and a ventilation fan on the chamber enclosure masked external noises.

In order to record the subjects' positions relative to the CS sources, the floor grids were connected to drinkometer circuits as in Experiment 1. For the present experiment, however, right and left floor sections were connected to "operate" leads from separate circuits. The center floor section, the chamber walls, and the chain were connected to the "common" leads of both circuits. The dipper-aperture was not equipped with a Plexiglas door and aperture entries were not recorded.

Procedure. Rats were trained to pull the chain to receive 3-sec access to a .1-ml dipper-cup of milk, and the schedule of milk deliveries was increased to a VI 2 min as in Experiment 1. When the rats had responded for 1045 -min sessions on this schedule, CS-only trials began. During each of 10 daily 15 -sec CS periods, occurring about once every $4.5 \mathrm{~min}$ (range $=2.5$ to $6.5 \mathrm{~min}$ ), one of the Plexiglas bars was lighted. Lighting of the right bar was the CS for one group (CS-US Near), while the left bar served as the CS source for the other group (CS-US Far). Positive conditioned suppression training began on the 11th day of CS presentations. During this CS-US pairings phase, three successive 3-sec operations of the milk dipper followed every CS. Thus, 
the groups differed according to the distance between the CSsource and US-delivery locations; distances between CS source and manipulandum and between US-delivery site and manipulandum were the same for all subjects. After 10 days of CS-US pairings, the CS-US Near subjects were switched to CS-US Far; during the last 15 days of the experiment, the left Plexiglas bar was the CS source for all subjects.

\section{Results and Discussion}

As in Experiment 1, daily US-approach ratios were computed from the amounts of pre-CS and CS time each subject spent contacting the right third of the floor. These ratios also reflect CS approach for the CS-US Near rats, because the near CS source was also on this side of the chamber. CS-approach ratios are not presented for CS-US Far subjects, because these rats rarely contacted the left floor section during pre-CS or CS.

Group US-approach ratios during CS-only and the first 10 days of CS-US pairings are compared in the left and center portions of Figure 3, where they have been averaged over two-session blocks. During the last three CS-only sessions, both groups spent about the same amount of pre-CS time contacting the right side of the floor $(t<1.0)$. However, CS-US Far subjects spent less time near the US-delivery site during CS than during pre-CS, whereas CS-US Near subjects spent more CS than pre-CS time contacting the right third of the floor. Group mean US-approach ratios for these sessions differed significantly [CS-US Near mean $=.56$, CS-US Far mean $=.44 ; \mathrm{t}(6)=3.82$, $\mathrm{p}<.01]$. This difference probably reflects unconditioned CS-approach responses.

During the 8th through 10th CS-US pairings sessions, both groups continued to spend nearly equal amounts of pre-CS time on the right side of the chamber $(t<1.0)$, and their US-approach ratios were again significantly different [CS-US Near mean $=.66$, CS-US Far mean $=.38 ; \mathrm{t}(6)=2.98, \mathrm{p}<.05$ ] Three of the four CS-US Far subjects had lower US-approach ratios for these sessions than for the last three CS-only sessions; this change probably reflects increases in behavior directed toward the far CS source in these subjects. All four CS-US Near subjects had higher US-approach ratios during the 8th through 10th CS-US pairings sessions than for the last three CS-only sessions $[\mathrm{t}(3)=3.26, \mathrm{p}<.05]$.

It was not possible to tell from these data, however, whether the conditioned response for CS-US Near subjects was CS approach or US approach, because both the CS source and the US-delivery site were on the right side of the front panel. Therefore, following the 10th CS-US pairings session, the CS-US Near subjects were switched to CS-US Far. If these subjects had been approaching CS rather than US, they might begin going to the left during the CS and their US-approach ratios would decrease. If, however, they had learned to approach the US-delivery

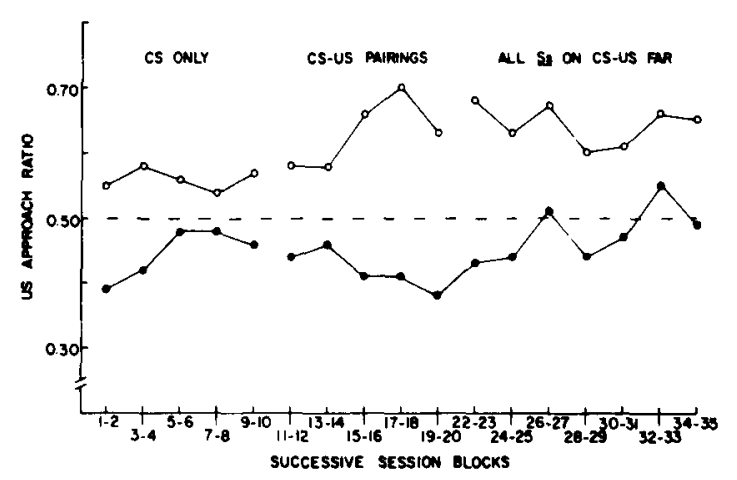

Figure 3. Mean US-approach ratios for CS-US Near (open circles) and CS-US Far (filled circles) subjects as a function of successive two-session blocks in Experiment 2.

site during CS, their US-approach ratios might show little change. The CS-US Far subjects were not switched to CS-US Near, but continued on the "far" arrangement to determine whether their US approach behavior would eventually be facilitated during CS relative to pre-CS.

As illustrated in the right portion of Figure 3, changing the CS location from near to far (i.e., from right to left) had little effect on these subjects' USapproach ratios. Neither their suppression ratios nor their US-approach ratios changed significantly from their last CS-US Near session to their first CS-US Far session (both ts $<1.0$ ); they all continued to approach the dipper during CS presentations when the condition was changed to CS-US Far. On the other hand, subjects trained on CS-US Far from the outset showed little tendency to approach the USdelivery site more during CS than during pre-CS even with extended training. Gross observations revealed that three of the four subjects in this group oriented toward (but rarely approached) their lighted bar CS on nearly every trial; all four of these subjects often alternated between orienting toward the CS source and approaching the dipper aperture during CS.

These data and observations suggest that, on the CS-US Near treatment, US approach accounts for positive conditioned suppression, whereas on the CS-US Far treatment, behavior directed toward the CS source plays a role in the disruption of responding during CS. Thus, CS-US proximity apparently is a determinant of which type of conditioned response develops. However, this experiment gives no information about the course of development of US-approach behavior in the CS-US Near subjects. It seems reasonable to suspect that CS-approach behavior may have predominated early in training for these subjects but given way to US-approach behavior, perhaps because dipper approaches were reinforced at the end of each CS.

The groups also differed on the suppression ratio measures, which are presented in Figure 4. As can be 
seen, chainpulling was asymptotically suppressed during CS in both groups after $10 \mathrm{CS}$-US pairings sessions. At asymptote, both groups pulled the chain at essentially the same rate during pre-CS $[\mathrm{t}(6)=$ 1.23 ], and the suppression ratios on the two arrangements did not differ (ts $<1.0$ ). However, a repeatedmeasures analysis of variance yielded a significant Sessions by Treatments interaction $[F(9,73)=4.91$, $\mathrm{p}<.011$, indicating that chainpulling was suppressed more rapidly in CS-US Near subjects than in CS-US Far subjects.

This result parallels that of Testa (1975), who found that suppression of leverpressing developed faster in rats when the CS source and an aversive (air-puff) US had similar locations than when they did not. Testa interpreted his result to mean that CS and US were more readily associated when they occurred in close spatial proximity to one another. This interpretation appears consistent with the present suppression data, but it cannot be applied unambiguously to them. According to a competing-response analysis, chainpulling in CS-US Near subjects was suppressed during CS because US-approach behavior was incompatible with this response. CS-US Far subjects, on the other hand, oriented toward their CS source more during CS than during pre-CS, and this response apparently interfered with their chainpulling. However, even though CS-US Far subjects spent less CS than pre-CS time near the US-delivery site, their US-approach ratios did not reach zero. This result is consistent with the observations of both orientation toward the CS source and dipper approaches in these subjects during the CS. Because the manipulandum was located between the CS source and the US-delivery site for these animals, their pattern of conditioned behavior actually kept them near the manipulandum during the CS and may have been responsible for the slower development of suppression on this arrangement. With continued CS-US pairings, "shuttling" back and forth between the CS and US apparently mediated suppression on the CS-US Far treatment.

The qualitative nature of these observations poses a problem for the analysis of suppression on the CS-US Far treatment. The observations are included mainly to suggest that the patterns of directed behaviors conditioned to the $\mathrm{CS}$ on positive conditioned suppression procedures may determine the rate at which suppression develops; Karpicke et al. showed similar patterns of directed behavior to determine the amount of positive conditioned suppression. The more quantitative data show CS-US proximity to be a variable influential in determining whether USapproach behavior is facilitated during positive conditioned suppression.

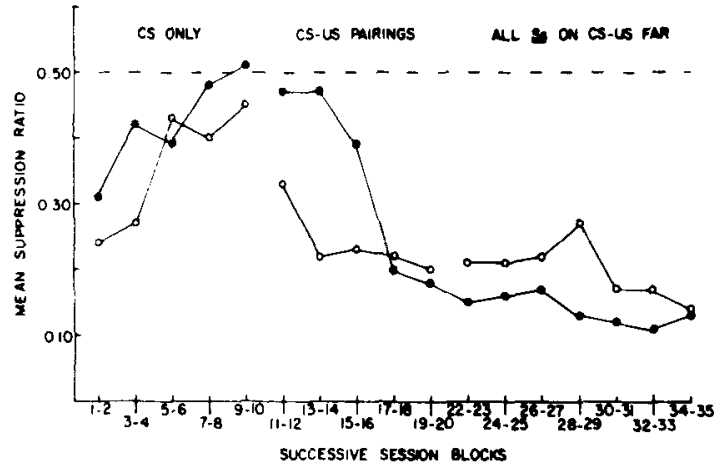

Figure 4. Mean chainpull ratios for CS-US Near (open circles) and CS-US Far (filled circles) subjects as a function of successive two-session blocks in Experiment 2.

\section{EXPERIMENT 3}

At this point, a role for directed responses in the production of positive conditioned suppression appeared to be established. However, the observed predominance of CS-directed behavior on the CS-US Far treatment in Experiment 2 seemed of possibly limited generality. For example, it seemed reasonable to predict that if the CS-source and US-delivery locations were far enough apart so that the rats would lose USs if they approached the CS, US approach might predominate. This prediction was based on studies which have arranged for contacts of a localized CS source to cause the omission of a scheduled US. Imposition of this omission contingency in autoshaping experiments with rat subjects decreases the number of contacts with the CS source (Boakes, 1977; Stiers \& Silberberg, 1974), and may increase the number of contacts with the US-delivery site during CS (Boakes, 1977). In Experiment 3, therefore, the "far" Plexiglas CS source was moved to the left side wall of the apparatus. This arrangement could impose an "implicit" omission-for-CS-approach contingency because approaches to this CS source location may cause subjects to lose USs.

\section{Method}

Subjects. Ten experimentally naive 100 - to 110 -day-old male hooded rats were reduced to $80 \%$ of their ad-iib weights. One subject was dropped from the experiment during the shaping procedure because it repeatedly jumped at the chain manipulandum and injured itself.

Apparatus. The apparatus was similar in all respects to the one used in Experiment 2, except that the right Plexiglas CS source had been removed and the left CS source was on the left wall about $2 \mathrm{~cm}$ from the interaction of the left and front walls, $7 \mathrm{~cm}$ above the grid floor. The distance between the chain and the closest edge of the Plexiglas bar was about $11 \mathrm{~cm}$, twice the distance between the CS and manipulandum in the CS-US Far condition of Experiment 2.

Procedure. Procedural details were the same as for CS-US 
Far subjects in Experiment 2. However, the rats received 30 rather than 25 CS-US pairings sessions.

\section{Results and Discussion}

During CS-US pairings, chainpulling by seven of the nine subjects was suppressed during CS relative to pre-CS. Chainpull suppression ratios, and USapproach and CS-approach ratios computed from the amounts of pre-CS and CS time spent on the right and left sides of the apparatus, respectively, are shown for these rats in Figure 5. Ratios for the two rats that did not show positive conditioned suppression are not presented. One of these rats rarely approached the CS source during CS or pre-CS. Its mean suppression and US-approach ratios for the last 3 days of training were .50 and .52 , respectively. The other rat eventually approached the CS source less during CS than during pre-CS, and its CS-approach ratio was .29 over the last 3 days of training. This subject's mean suppression and US-approach ratios for these days were .51 and .50 , respectively.

All of the seven rats that showed positive conditioned suppression approached the CS source more during CS than during pre-CS in the CS-only phase and early in the CS-US pairings phase. Even though CS-approach ratios during CS-only were high, they reflected very small amounts of time spent contacting the left floor section (pre-CS mean $=.5 \mathrm{sec}$, CS mean $=1.1 \mathrm{sec}$, for the final three CS-only sessions). For this reason, they probably do not reflect increases in behavior which could compete strongly with chainpulling during CSs. With continued CS-US pairings, CS-approach ratios decreased. Eventually, all rats approached the CS source less during CS than during pre-CS, and approached the US-delivery site more during CS than during pre-CS. Over the last three CS-US pairings sessions, their mean suppression ratio was .24, their mean US-approach ratio was .61 , and they had a mean CS-approach ratio of .34 . Each of these means differed significantly from a .50 baseline ratio $[\operatorname{ts}(6)>3.91$, ps $<.01]$.

Approaching the far CS location in this experiment may have prevented efficient collection of USs and thereby produced the increases in US-approach ratios and decreases in CS-approach ratios which developed with repeated CS-US pairings. This suggestion is consistent with Boakes' (1977) report that an omissionfor-CS-contact contingency caused rats to switch from contacting the CS source to contacting the USdelivery site during CSs.

It is unfortunate that the numbers of "lost" USs were not recorded in this experiment; these data could make the "implicit" omission contingency explanation more than a speculation. However, this experiment, in conjunction with Experiment 2, does suggest that CS-source and US-delivery locations on positive conditioned suppression procedures determine

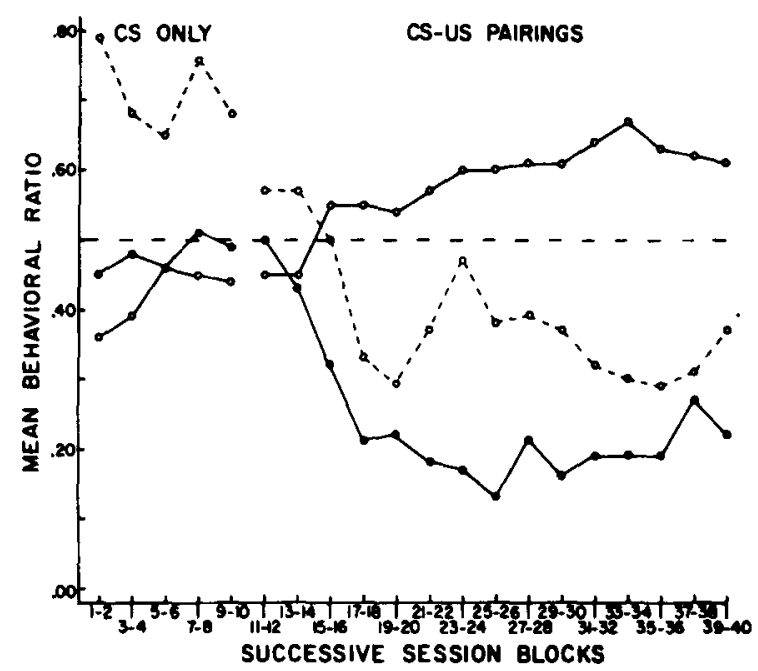

Figure 5. Mean chainpull ratios (filled circles), US-approach ratios (open circles, solid lines), and CS-approach ratios (open circles, broken lines) for the seven rats that showed positive conditioned suppression in Experiment 3.

whether CS-directed or US-directed responses will predominate during the CS. Although this suggestion is based in part on a between-experiment comparison, it seems likely that future analyses will find that subtle features of these arrangements are responsible for the relative predominance of one or the other response.

\section{GENERAL DISCUSSION}

This series of experiments showed that directed skeletal responses conditioned to preappetitive CSs accompany positive conditioned suppression and presumably interfere with instrumental responding during CS presentations. In Experiment 1, the amount of interference with ongoing behavior during the CS appeared to depend upon whether USs and instrumental reinforcers had the same or separate delivery locations. More positive conditioned suppression occurred with the latter than with the former arrangement. In Experiment 2, US-approach responses appeared to predominate when CS and US locations were very close together, whereas orientation toward the CS source and "shuttling" between CS and US locations apparently interfered with instrumental responding when the CS-source and US-delivery device were on opposite sides of the manipulandum. When the CS-source and US-delivery locations were far enough apart so that approach to the CS source might have interfered with collection of USs, US approach also predominated (Experiment 3 ).

The discussion of the data obtained in Experiment 3 was based on Boakes' (1977) report that when CS contacts resulted in the omission of USs, CS contacts decreased and contacts with the US-delivery 
device increased during the CS. Boakes found, however, that without this contingency some subjects showed mainly CS approach and others showed mainly US approach on the same spatial arrangement of CS-source and US-delivery site. In the present experiments, response predominance was more consistent from subject to subject on a given spatial arrangement.

Positive conditioned suppression procedures and autoshaping procedures such as Boakes' differ in a potentially important way, however. With positive conditioned suppression procedures, the use of an instrumental-response manipulandum largely determines the position of the subject relative to the CS and US locations at the beginning of each trial, whereas with autoshaping procedures a subject's position relative to CS and US is not experimentally controlled. The facts that similar conditioned behaviors developed in most subjects in any given proce dure in the present experiments and that some of Boakes' subjects performed one response and some another suggest that the subject's proximity to the CS-source and US-delivery locations at trial onset may be an important determinant of whether CS approach or US approach predominates. The relationships among CS source, US-delivery site, and the subject's position in the apparatus at trial onset have not, however, been systematically investigated in either autoshaping or positive conditioned suppression procedures. These variables merit further attention.

It is difficult to determine why CS-approach and US-approach behaviors have not been reported in other investigations of positive conditioned suppression. However, only two of these studies (Azrin \& Hake, 1969; Leslie \& Millenson, 1973) have either reported observations of their subjects or attempted to measure these kinds of behaviors.

Although Azrin and Hake do not give a very detailed description of their apparatus, it seems likely that, because they used several types of CSs and USs, they employed several CS-source and US-delivery locations. This variation could, as the present studies suggest, prevent the development of consistent patterns of behavior across experiments. Thus, their gross observations may not have been sensitive enough to detect whatever common properties the conditioned behaviors had from experiment to experiment. Although some of the observations in the present experiments were also qualitative, the quantitative measures showed directed skeletal responses to emerge quite reliably.

Leslie and Millenson, however, attempted to measure US-approach behavior during positive conditioned suppression but found no increase in foodtray entries during CSs. At least three features of their experiment seem potentially responsible for this result. First, the click stimulus they used as a CS may have been localizable enough to permit conditioning of CS approaches; these responses would have competed both with leverpressing and with approaching the food tray. Second, although there was a "door" in front of the food tray, it was not clear from their description of this device how far the door had to be pushed open to close the microswitch that recorded tray entries. It is possible that behavior was directed at this device without being recorded. This suggestion seems plausible in light of the third, and possibly most important, difference between their experiment and those that have found US-approach behavior to emerge during positive conditioned suppression: The food pellets they used as USs were available until the rat ate all of them, whereas in the present experiments, and in Karpicke et al., appetitive USs would be lost if the rats did not act promptly to secure them. Thus, there is reason to believe that approaches to the US-delivery site on the Leslie and Millenson procedure were less vigorous than with the type of US-delivery procedure used in the present study. These less vigorous responses may not have been measurable by their apparatus.

The measurements obtained in the present experiments suggest, however, that CS-approach and USapproach responses are quite reliable phenomena during positive conditioned suppression. They further suggest that the topographies of conditioned responses on any positive conditioned suppression procedure depend upon the spatial arrangements of features which elicit and support these behaviors. The identification of these features, and the spatial relationships between them which will support responses with differing topographies, appears necessary for the analysis of appetitive classical-instrumental interactions.

\section{REFERENCES}

Azrin. N. H., \& HaKe, D. F. Positive conditioned suppression: Conditioned suppression using positive reinforcers as the un. conditioned stimuli. Joumal of the Experimental Analysis of Behavior, 1969, 12, 167-173.

BOAKES. R. A. Performance on learning to associate a stimulus with positive reinforcement. In $\mathrm{H}$. Davis \& H. Hurwitz (Eds.) Operant-Pavlovian interactions. Hillsdale, N.J: Erlbaum, 1977.

Estes, W. K., \& Skinner, B. F. Some quantitive properties of anxiety. Journal of Experimental Psychology. 1941, 29, 390-400.

HeArst, E., \& Jenkins, H. M. Sign-tracking: The stimulusreintorcer relation and directed action. Austin, Texas: Psychonomic Society, 1974.

Holland, P. C. Conditioned stimulus as a determinant of the form of the Pavlovian conditioned response. Joumal of Experimental Psychology: Animal Behavior Processes, 1977, 3, 77-104.

Karpicke. J.. Christoph, G.. Peterson, G., \& Hearst. E Signal location and positive vs. negative conditioned suppression in the rat. Joumal of Experimental Psychology: Animal Behavior Processes. 1977. 3. 105.118. 
KeLLY, D. D. Long-term prereward suppression in monkeys unaccompanied by cardiovascular conditioning. Joumal of the Experimental A nalysis of Behavior, 1973, 20, 93-104.

Leslie, J., \& Mrtuenson, J. R. Effects of signaled free reinforcement on concurrent performances. Bulletin of the Psychonomic Society, 1973, 1, 97-99.

LoLordo, V. M., McMmLan, J. C., \& RnEY, A. L. The effects upon food-reinforced pecking and treadle-pressing of auditory and visual signals for response-independent food. Learning and Motivation, 1974, 5, 24.41.

Millenson, J. R., \& DeVilliers, P. A. Motivational properties of conditioned anxiety. In R. M. Gilbert \& J. R. Millenson (Eds.), Reinforcement: Behavioral analysis. New York: Academic Press, 1972.

Rescorla, R. A., \& Solomon, R. L. Two-process learning theory: Relationships between Pavlovian conditioning and instrumental learning. Psychological Review, 1967, 74, 151-182.
SChwartz, B. Positive and negative conditioned suppression in the pigeon: Effects of the locus and modality of the CS. Learning and Motivation, 1976, 7, 86-100.

Stiers, M., \& Silberberg, A. Lever-contact responses in rats: Auto-maintenance with and without a negative responsereinforcer dependency. Journal of the Experimental Analysis of Behavior, 1974, 22, 497-506.

Testa, T. J. Effects of similarity of location and temporal intensity pattern of conditioned and unconditioned stimuli on the acquisition of conditioned suppression in rats. Journal of Experimental Psychology: Animal Behavior Processes, 1975, 1. 114-121.

(Received for publication July 26, 1977; revision accepted January 3.1978.) 